\title{
AML-6-Studie zum Wert einer zyklisch alternierenden Chemotherapie während der Remission bei akuter myeloischer Leukämie
}

\begin{tabular}{|l|l|l|}
\hline U. & Jehn $^{\text {a }}$ \\
\hline R. & Zittoun $^{\text {b }}$ \\
\hline
\end{tabular}

${ }^{\mathrm{a}}$ Med. Klinik III, Klinikum Großhadern, München ${ }^{\mathrm{b}}$ Service d'Hématologie, Hotel Dieu, Paris

\section{Schlüsselwörter}

Akute nicht-lymphatische Leukämie

Zyklisch alternierende Chemotherapie

Rezidiv-Rate

\section{Zusammenfassung}

25 Kliniken nehmen an einer randomisierten Studie der E.O.R.T.C. zur Verbesserung der Remissionsrate, der Remissionsdauer und der Überlebenszeit bei der akuten nicht-lymphatischen Leukämie im Erwachsenenalter teil. Um den Zeitpunkt des Rezidivs nach erreichter Remission hinauszuzögern, wird eine intensive, zyklisch alternierende Behandlung nach einer frühen Konsolidierung durchgeführt, welche entweder dieselben Medikamente der Induktionstherapie oder abwechselnd Kombinationen mit anderen Zytostatika enthält wie mAMSA, 5-AZA und HDAra C. Bisher sind 266 Patienten in die Studie eingebracht worden. Das mittlere Alter beträgt 45 Jahre. Die Remissionsrate liegt bei $71 \% ; 52 \%$ der Responder erreichten diese nach einem Tnduktionszyklus. $10 \%$ der Patienten starben entweder innerhalb von 7 Tagen nach Behandlungsbeginn oder danach an einer Hypoplasie; 13\% erwiesen sich als Therapie-resistent. Bisher sind 112 Patienten für die «Erhaltungstherapie» randomisiert worden, 58 in den Arm I und 54 in Arm II, 79/112 befinden sich noch in der Behandlung. Die Toxizität des Therapie-Protokolls ist vertretbar: Sie war bisher bei $7 \%$ Grund für einen Therapie-Abbruch während der Induktion und bei 3\% während der «Erhaltung». Über Remissionsdauer und Überlebenszeit in beiden Therapie-Armen können noch keine Aussagen gemacht werden. Sonderdruckbestellungen an: Prof. Dr. U. Jehn, Med. Klinik III, Klinikum Großhadern, Marchioninistr. 15, D-8000 München
70

Summary and Key Words

Twenty-five institutions are participating in a randomized trial of the E.O.R.T.C. for improvement of remission incidence, disease-free interval and survival in adult acute nonlymphocytic leukemia. In order to delay the time of relapse, an intensive cyclic therapy is employed early after achievement of complete remission (C.R.) using either the same drugs of the induction regimen or rotating combinations of alternative drugs, e.g. mAMSA, 5-AZA and HD-Ara C. So far 266 patients entered the trial with a median age of 45 yrs. The overall C.R. rate is $71 \% ; 52 \%$ of the responders reached C.R. after 1 cycle of induction treatment. $10 \%$ of the patients died within the first 7 days of induction or due to hypoplasia, 13\% were absolute resistant to this regimen. Fifty-eight patients are randomized to 'maintenance' arm I, 54 to arm II, 79/112 patients are still under study. Treatment toxicity is tolerable. In $7 \%$ and $3 \%$ excessive toxicity was a reason for going off study during induction or 'maintenance'. As far as remission duration or survival within the two treatment arms is concerned, it is too early to draw any conclusions.

Acute non-lymphocytic leukemia - Cyclic alternate chemotherapy -Relapse rate 
Wissenschaftlicher Hintergrund und Fragestellung

Die Remissionsraten nach Behandlung der AML liegen zwi-schen 60 und 70\%, in einigen Zentren sogar bei $80 \%$. Auch die 5 aufeinanderfolgenden, multizentrischen EORTC-Stu-dien haben eine deutliche Verbesserung von 16 auf $60 \%$ erbracht. Gleichzeitig konnten in den letzten Jahren die Remissionsdauer, welche je nach Studie 40 und 50 Wochen beträgt, sowie die Überlebenszeit, die im Mittel bei 2 Jahren liegt, verbessert werden.

Dennoch erleiden 80-90\% der erfolgreich behandelten Patienten 3 Jahre nach Diagnosestellung einen Rückfall. Einige randomisierte Studien haben unter Verwendung der konventionellen Erhaltungstherapie keine Verlängerung des krankheitsfreien Intervalls gebracht. Ebensowenig hat die AML-5-Studie weder durch Hinzufügen einer Immuntherapie noch von Androgenen einen erkennbaren Vorteil gezeigt. Vielmehr scheinen die Verbesserungen der Remissionsdauer und der Überlebenszeit einiger Studien auf einer Intensivie-rung der initialen Induktionstherapie mit anschließender, kur-zer Konsolidierung zu beruhen.

1 Teilweise vorgetragen bei der 29. Jahrestagung der Deutschen und Österreichischen Gesellschaft für Hämatologie und Onkologie vom 7.-10.10.1984 in Ulm.

Faktoren, welche möglicherweise für den Rückfall verant-wortlich sind, könnten in einer inadäquaten primären Zytore-duktion oder in der Entstehung einer Resistenz auf die verwendeten Zytostatika bestehen. Um diese Frage zu beantwor-ten und damit das Rezidiv hinauszuschieben und eventuell auch die Überlebenszeit zu verlängern, wird in der AML-6Studie zwischen einer zyklischen «Erhaltungstherapie» unter Verwendung neuer Substanzen in alternierendem Wechsel, die nicht zur Induktion und Konsolidierung verwendet wur-den, und der mehrfach zu wiederholenden initialen Induk-tionszyklen in abgeschwächter Dosierung randomisiert. Wei-tere Fragen sind in dieser Studie auf die Toxizität und Lebens-qualität in beiden remissionserhaltenden Armen gerichtet.

Beteiligte Kliniken, Studienplan, Einschlußkriterien

Die beteiligten 25 Zentren sind am Schluß des Beitrages zusammengestellt, aus der BRD beteiligen sich Köln und München, andere deutsche Kliniken sind eingeladen, sich an dieser noch offenen Studie oder Folgestudien zu beteiligen. Der Studienplan ist in Abbildung 1 dargestellt: Daunorubicin wird an 3 aufeinanderfolgenden Tagen gegeben, Cytosin-Arabinosid über 7 Tage, und zwar sowohl als Dauerinfusion als auch zusätzlich - aufgrund der guten Erfahrung aus der Jehn, Zittoun: E.O.R.T.C. - AML-6-Studie 95

AML-6

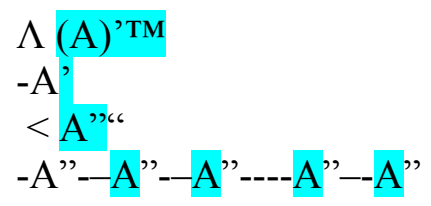


$-\mathrm{C}-\mathrm{B} \quad \mathrm{C} \quad \mathrm{B} \quad \mathrm{C}$

Wo 44

66

6666

Induktion:

A

$=\mathrm{DNR}$

$45 \mathrm{mg} / \mathrm{m} 2$ i.v. Tag 1,2,3

VCR

$1 \mathrm{mg} / \mathrm{m} 2$ i.v. Tag 2

\section{AraC}

$100 \mathrm{mg} / \mathrm{m} 2$ Dauerinfusion

Tag 1-7 $50 \mathrm{mg} / \mathrm{m} 2$ i.v. push q $12 \mathrm{~h}$

(A)

= Wiederholung von A wenn keine VR

Konsolidierung:

A'

$=\mathrm{DNR}$

$45 \mathrm{mg} / \mathrm{m} 2$ i.v. Tag 1

VCR

wie in $A$

AraC

wie in $\mathrm{A}$

Erhaltung:

A"

$=\mathrm{DNR}$

wie in A'

VCR

wie in $A^{\prime}$

AraC

$100 \mathrm{mg} / \mathrm{m} 2$ s.q. q $12 \mathrm{~h}$ Tag $1-5$ 
$\mathrm{B}$

$=$ AMSA

$150 \mathrm{mg} / \mathrm{m} 2$ i.v. Tag 1

AraC

$3 \mathrm{mg} / \mathrm{m} 2$ i.v. q $12 \mathrm{~h}$ Tag 1,2

$\mathrm{C}$

$=$ AMSA

$150 \mathrm{mg} / \mathrm{m} 2$ i.v. Tag 1

5-AZA

$150 \mathrm{mg} / \mathrm{m} 2$ i.v. Tag $1,2,3$

Abb.l. Studienplan.

Ziele der Studie: Hinauszögern des Rezidivs. E.O.R.T.C.-06821

vorangegangenen AML-5-Studie im Hinblick auf die präven-tive ZNS-Wirkung - als Bolus alle 12 h. Nach Eintreten einer Vollremission erfolgt unmittelbar anschließend eine Konsolidierung mit dem gleichen Induktionszyklus, jedoch Daunoru-bicin auf Tag 1 beschränkt. Die beiden remissionserhaltenden Arme bestehen zum einen aus der 6-maligen Wiederholung des

Konsolidierungs-Schemas in 6-wöchigem Abstand, wobei Ara C subkutan verabfolgt und auf 5

Tage verkürzt wird. Zum anderen wird im 2. Arm zyklisch alternierend mAMSA zusammen mit hochdosiertem Ara $\mathrm{C}$ an 2 Tagen bzw. mit 5-Azacytiden an 3 Tagen jeweils 3x gegeben. Um die Frage einer möglichen Resistenzentwicklung weiter zu untersuchen, wird bei Auftreten eines Rezidivs nach vollstän-diger, abgeschlossener «Erhaltungstherapie», bestehend aus 6 Zyklen, folgendermaßen verfahren: Rezidive nach Abschluß des Armes «A» oder I werden mit mittelhoch-dosiertem und hochdosiertem Ara C zusammen mit mAMSA behandelt (EORTCProtokoll 06841), Rezidive nach dem Arm B-C oder II mit demselben Induktions-Schema, welches initial verwendet wurde. Einschlußkriterien für die Studie sind alle neu diagnostizierten, nicht vorbehandelten AML-Patienten zwischen 10 und 65 Jahren aller 6 zytologischen Typen entspre-chend der FAB-Klassifikation, vorausgesetzt, daß sie $>30 \%$ Blasten im Knochenmark haben. Ausgeschlossen werden Patienten mit CML-Blastenkrise, Leukämien nach myeloproliferativen oder myelodysplastischen Syndromen, Leukämien nach zytostatischer bzw.

immunsuppressiver Behandlung oder bei Zweittumoren sowie Patienten mit schweren Herz-, Nie-ren- oder Lebererkrankungen.

Bisherige Ergebnisse

Bisher wurden 266 Patienten in die Studie eingebracht. Das mittlere Alter beträgt 45 Jahre. Die Remissionsrate liegt bei

$71 \%$, wobei $52 \%$ der Responder bereits nach dem 1. Zyklus in eine Vollremission kamen. 2 Patienten $(=0,8 \%)$ erreichten nach zwei Induktionszyklen eine Teilremission. Die Frühtodesrate, definiert als Tod innerhalb der ersten 7 Tage nach Therapiebeginn, ist 3,3\%, die Todesrate aufgrund einer Kno-chenmarkaplasie im gesamten Zeitraum der Induktionsphase 6,7\%. Eine persistierende Hypoplasie nach Abschluß der Induktionsbehandlung wurde in 0,4\% der Fälle gesehen, eine Hypoplasie, welche von erneutem leukämischem Wachstum gefolgt war, in der Größenordnung von 5\%. Eine absolute Therapieresistenz bestand bei etwa 13\% der 
Patienten. Zwischen den verschiedenen zytologischen Untergruppen entspre-chend der FABKlassifikation wurde kein Unterschied in der Ansprechrate gefunden. Ebensowenig hatten die Anzahl der initialen Blasten im Knochenmark, die initialen peripheren Leukozytenwerte oder das Alter eine prognostische Relevanz auf die Remissionsrate. Wie schon in der AML-5-Studie [1], ist offenbar ein hoher Prozentsatz von Auer-Stäbchen ( $>2,5 \%$ bei $200 \mathrm{KM}-Z$ ellen) prognostisch günstig. 112 Patienten erreichten bisher die Randomisierung in die beiden Arme der «Erhaltung»: 58 in den Arm I und 54 in Arm II; erst bei 4 Patienten ist diese vollständig beendet worden. Bei 79/112 Patienten ist dieser Behandlungsabschnitt noch nicht beendet. 18/112 Patienten bekamen jetzt ein Rezidiv während der «Er-haltungsphase».

Die Verträglichkeit der Therapie ist erstaunlich gut, die Toxizi-tät tolerabel. Toxizität als Grand für Therapieabbruch wurde bisher bei 7\% während der Induktion und 3\% während der «Erhaltung» angeführt. Da die Beobachtungszeit, insbeson-dere nach vollständig abgeschlossener «Erhaltungstherapie» noch zu kurz und die Fallzahlen für diesen Therapieabschnitt zu gering sind, können noch keine Aussagen über Remissions-dauer oder Überlebenszeit, geschweige denn getrennt für beide Arme - dem eigentlichen Ziel dieser Studie - gemacht werden. Über eine Beteiligung weiterer deutscher Zentren würden wir uns freuen. Beteiligte Institutionen

Belgien: Inst. J. Bordet (Brüssel), Saint-Pierre (Brüssel), Hop. de Bavière (Lüttich), St. Jan's Hosp. (Brugge), AZ (Antwerpen), Hop. de Verviers (Verviers). C.G.T.R. (Montige Le Tilleul) BRD: Univers.-Kl. (Köln), Klinikum Großhadern (München) Frankreich: Henri Becquerel (Rouen), C.A. Lacassagne (Nizza), Ins. Gust. Roussy (Villejuif), Fond Bergonie (Bordeaux), Hop. Ed. Her-riot (Lyon), Hotel-Dieu (Paris), Hop. Bellevue (Bordeaux) Holland: Groot Ziekengast (sHertogenbosch), R.R.T.J. (Rotterdam), St. Radbout Ziek (Nijmegen), Olv' Gast (Amsterdam), AZ Leiden (Leiden), Leyenburg (Den Haag), Erasmus Univ. (Rotterdam). Italien: Osp. Maggiore (Mailand) Schweden: Karolinska Sjukh (Stockholm)

Literatur 1 Zittoun, R.; Cadiou, M.; Bayle, Ch,; Suciu, St.; Solbu, G.: Prognostic value of cytologic parameters in acute myelogenous leukemia. Cancer 53: 1526-1532 (1984). 